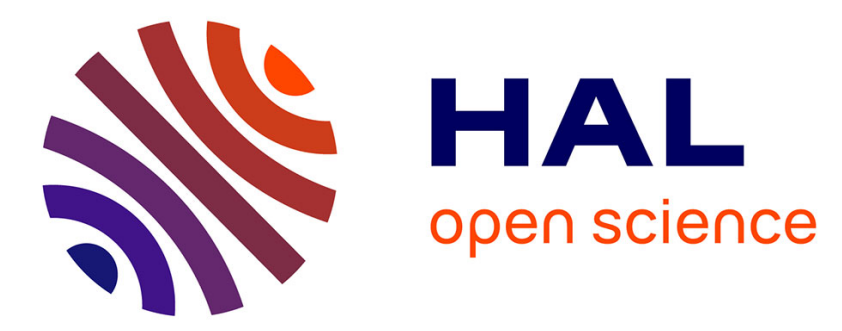

\title{
Charge-spin current conversion in high quality epitaxial Fe/Pt systems: Isotropic spin Hall angle along different in-plane crystalline directions
}

\author{
C Guillemard, S. Petit-Watelot, S. Andrieu, J.-C. Rojas-Sánchez
}

\section{To cite this version:}

C Guillemard, S. Petit-Watelot, S. Andrieu, J.-C. Rojas-Sánchez. Charge-spin current conversion in high quality epitaxial Fe/Pt systems: Isotropic spin Hall angle along different in-plane crystalline directions. Applied Physics Letters, 2018, 113, 10.1063/1.5079236 . hal-02999961

\section{HAL Id: hal-02999961 \\ https://hal.univ-lorraine.fr/hal-02999961}

Submitted on 11 Nov 2020

HAL is a multi-disciplinary open access archive for the deposit and dissemination of scientific research documents, whether they are published or not. The documents may come from teaching and research institutions in France or abroad, or from public or private research centers.
L'archive ouverte pluridisciplinaire HAL, est destinée au dépôt et à la diffusion de documents scientifiques de niveau recherche, publiés ou non, émanant des établissements d'enseignement et de recherche français ou étrangers, des laboratoires publics ou privés. 


\title{
Charge-spin current conversion in high quality epitaxial Fe/Pt systems: Isotropic spin Hall angle along different in-plane crystalline directions
}

\author{
C. Guillemard, S. Petit-Watelot, ${ }^{\text {a) }}$ S. Andrieu, and J.-C. Rojas-Sánchez ${ }^{\text {b) }}$ \\ Université de Lorraine, CNRS UMR 7198, Institut Jean Lamour, F-54011 Nancy, France
}

(Received 29 October 2018; accepted 12 December 2018; published online 28 December 2018)

\begin{abstract}
We report the growth of $\mathrm{MgO}[001] / / \mathrm{Fe}(6 \mathrm{~nm}) / \mathrm{MgO}(7 \mathrm{~nm})$ and $\mathrm{MgO}[001] / / \mathrm{Fe}(6 \mathrm{~nm}) / \mathrm{Pt}(6 \mathrm{~nm})$ by molecular beam epitaxy and show that the full characterization by spin-orbit ferromagnetic resonance (SO-FMR) allows the determination of magnetic anisotropies by classical FMR-only studies. The spin mixing conductance of the epitaxial Fe/Pt interface was measured to be $g_{\text {effect }}^{\uparrow \downarrow}=2.6 \pm 0.5 \times 10^{19}$ $\mathrm{m}^{2}$, and the effective spin Hall angle (SHA) $\theta_{\mathrm{SHE}}^{\text {effect }}$ was estimated at different in-plane crystalline directions. It was found that $\theta_{\mathrm{SHE}}^{\text {effect }}$ is the same in all directions. When taking into account high enough excitation frequencies to achieve uniform precession of magnetization, the effective SHA for epitaxial $\mathrm{Pt}$ in $\mathrm{Fe} / \mathrm{Pt}$ is $\theta_{\mathrm{SHE}}^{\text {effect }}=0.051 \pm 0.005$. We address about the proper conditions to determine those relevant spintronic parameters. Published by AIP Publishing. https://doi.org/10.1063/1.5079236
\end{abstract}

The conversion of spin current into charge current and vice versa plays key roles in new research efforts in spintronics and related applications. This interconversion can be achieved without any external magnetic field or magnetic material in 3-dimensional systems that exhibit strong spinorbit coupling, ${ }^{1}$ namely, the spin Hall effect (SHE). ${ }^{2-4}$ The quantification of the efficiency of such interconversion is called the spin Hall angle (SHA). The spin Hall angle determination is thus relevant to find out new materials for applications like in magnetic memories because it will allow reducing power consumption. Large spin Hall angles have been found in heavy metals like $\mathrm{Pt},{ }^{5-8} \mathrm{Ta},{ }^{9}$ and $\mathrm{W}^{10,11}$ and alloys like $\mathrm{CuBi},{ }^{12} \mathrm{AuW}$, and AuTa. ${ }^{13,14}$ In a heavy metal or alloy layer which is in contact with a layer of a different material, an injected spin current might decrease through the interface due to interfacial interactions and the spin Hall angle becomes an effective spin Hall angle $\theta_{\mathrm{SHE}}^{\text {effect }} ., 15,16$

There are several techniques available to evaluate the effective spin Hall angle or effective spin orbit torque (SOT), like spin pumping ferromagnetic resonance, ${ }^{7,17-20}$ spin-orbit ferromagnetic resonance (SO-FMR), ${ }^{6,21-24}$ no-local injection in lateral spin valves, current-induced magnetization switching, harmonic measurements, spin Hall magnetoresistance, and so on. In all these experiments, in the measurements as well as in the analysis, there are many details and approximations that are often overlooked. So far, the SHA quantification has been evaluated mostly on sputtered polycrystalline samples. There is a lack of such experimental evaluation in epitaxial samples considering different crystalline axes. In this paper, we focus on the spin-orbit or spin-torque ferromagnetic resonance (SO-FMR, ST-FMR) technique to study epitaxial samples. We have patterned bars along different crystallographic directions. The results show isotropic values for the thickness level in this study, $\mathrm{Fe}(6 \mathrm{~nm}) / \operatorname{Pt}(5 \mathrm{~nm})$.

Epitaxial $s / / \mathrm{Fe}(6 \mathrm{~nm}) / \mathrm{Pt}(5 \mathrm{~nm})$ bilayers were grown by molecular beam epitaxy, where $s$ stands for the crystalline [001] MgO substrate.

\footnotetext{
a) sebastien.petit@univ-lorraine.fr

b) juan-carlos.rojas-sanchez@univ-lorraine.fr
}

The charge-spin current conversion was evaluated by SO-FMR. For comparison, a single Fe layer capped with $7 \mathrm{~nm}$ of $\mathrm{MgO}$ was grown and characterized by the classical FMR method with magnetic field $H_{\mathrm{dc}}$ applied in-plane along different crystalline directions.

The Fe layer was grown simultaneously for the $s / / \mathrm{Fe} /$ $\mathrm{MgO}$ reference sample as for the $s / / \mathrm{Fe} / \mathrm{Pt}$ bilayer as shown in Fig. 1. After the deposition of the Fe layer, half of the sample was covered to deposit $7 \mathrm{~nm}$ of $\mathrm{MgO}$, and then, the operation is reversed to deposit the Pt layer. In such a way, we have a true Fe reference layer to estimate, for instance, the effective spin mixing conductance at the $\mathrm{Fe} / \mathrm{Pt}$ interface. The $2 \mathrm{D}$ growth and quality of the sample were monitored in-situ by reflection high-energy electron diffraction (RHEED) as shown in Fig. 1(b). The Fe crystalline cell grown rotated by $45^{\circ}$ on top of the $[001] \mathrm{MgO}$ crystalline cell because of their lattice parameters, that is, the direction $[110] \mathrm{Fe} \|[100] \mathrm{MgO}$ and the direction [100]Fe $\|[110] \mathrm{MgO}^{25}$ In the following, we will refer only to the $\mathrm{MgO}$ crystalline axes.

Small pieces of about few $\mathrm{mm}^{2}$ were extracted from the center region of the $s / / \mathrm{Fe} / \mathrm{MgO}$ sample to avoid edge deposition issues and measured by FMR. A grounded coplanar wave guide (GCPW) was used as shown in Fig. 2(a). Hence, the

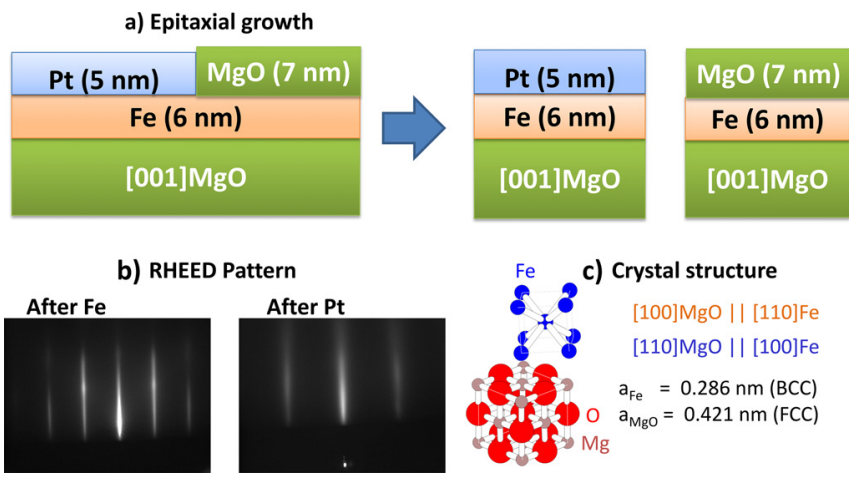

FIG. 1. (a) Schematic of the full stack that has been grown by MBE. After the deposition of the Fe layer, half of the sample was cover to deposit $7 \mathrm{~nm}$ of $\mathrm{MgO}$ or $5 \mathrm{~nm}$ of Pt. (b) RHEED pattern showing the good quality and 2D growth of Fe as well as the Pt layers. (c) The cubic Fe cell grown rotated by $45^{\circ}$ on top of the cubic cell of $\mathrm{MgO}$. 
radio-frequency magnetic field was maintained transversal (along the $y$ direction) to the dc magnetic field $H_{\mathrm{dc}}$ (along $x$ ). The DC field was applied parallel to the film plane in the present study. The frequency and input power of the microwave source were fixed, and $H_{\mathrm{dc}}$ was swept around the resonance field as shown in Fig. 2(b). The transmitted power is detected using a power diode detector and the lock-in technique. The amplitude of the $\mathrm{rf}$ power was modulated at $f_{\mathrm{LO}}(=433 \mathrm{~Hz})$, and the output signal of the diode is measured at $f_{\mathrm{LO}}$. A typical FMR spectrum is shown in Fig. 2(b) when $H_{\mathrm{dc}}$ is applied parallel to the $[100] \mathrm{MgO}$ crystalline axis. The observed symmetrical Lorentz curve verifies that, in this geometry, the absorbed microwave power is proportional to the imaginary part of the magnetic susceptibility $\chi_{y y}{ }^{\prime \prime}$ with practically no losses due to dispersion in the GCPW. We can identify the resonance field $H_{\text {res }}$ and the linewidth $\Delta H$ (half width at half maximum). Broadband frequency dependence was studied when $H_{\mathrm{dc}}$ is applied along different crystalline directions. The $f$-dependence of the resonance field and linewidth was analyzed and is plotted in Figs. 2(c) and 2(d), respectively. We can observe in Fig. 2(c) the typical relationship dispersion $f$ vs. $H$ in a system with cubic magnetocrystalline anisotropy where the in-plane easy axes are along the $[110] \mathrm{MgO}$ directions and the in-plane hard axes are along [100] MgO ones. It is worth noting that for every $90^{\circ}$, we recover the same relationship without any shift (neither vertical nor horizontal). This means the lack of additional in-plane uniaxial anisotropy, which is an experimental verification of a high quality sample. After minimizing numerically the free density energy of the system, we get the equilibrium position of the magnetization and the resonance condition dispersion following the results a) FMR setup

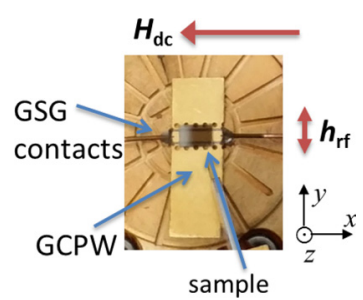

c) $f$ vs $H$

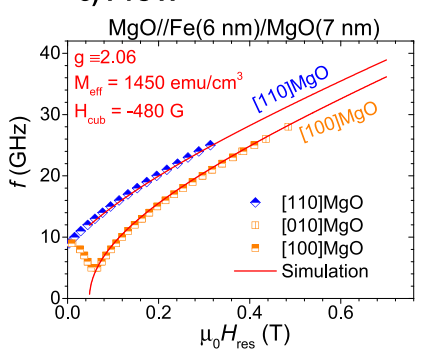

b) FMR spectrum

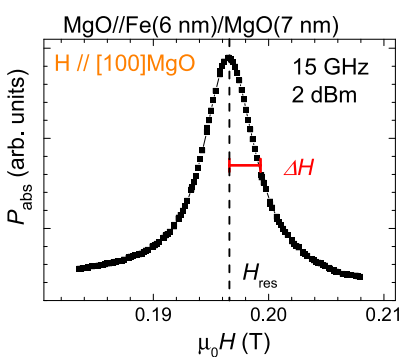

d) $\Delta \boldsymbol{H}$ vs $\boldsymbol{f}$

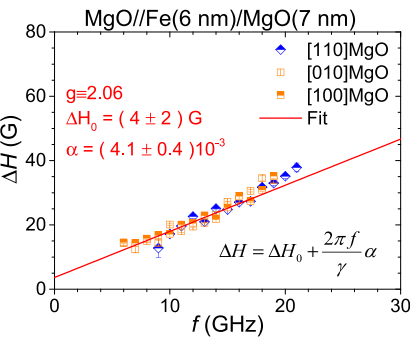

FIG. 2. (a) Picture of the grounded coplanar wave guide (GCPW) used along with grounded-signal-grounded (GSG) radiofrequency contacts. The sample position, the direction of the dc magnetic field, $\mathrm{H}_{\mathrm{dc}}$, and the direction of the rf field, $h_{\mathrm{rf}}$, in the middle of the signal line are also indicated. (b) Typical FMR spectrum. One can identify the resonance field $\mathrm{H}_{\text {res }}$ and the linewidth $\Delta \mathrm{H}$ for a frequency of $15 \mathrm{GHz}$ when $\mathrm{H}$ is applied parallel to the [100] $\mathrm{MgO}$ crystalline direction. (c) Dispersion relationship f vs $\mathrm{H}_{\text {res. }}$ We can identify the easy, $[110] \mathrm{MgO}$, and in-plane hard axes, $[100] \mathrm{MgO}$, due to cubic magnetocrystalline anisotropy. Lines are numerical simulation as described in the text. (d) Determination of magnetic damping constant $\alpha$. of Smit and Beljers ${ }^{26}$ and Suhl, ${ }^{27}$ whose formalism has been developed in detail elsewhere. ${ }^{28}$ Thus, the effective magnetic saturation $M_{\text {eff }}$ is $1450 \mathrm{emu} / \mathrm{cm}^{3}=1450 \mathrm{kA} / \mathrm{m}$, and the cubic magnetocrystalline field $\mu_{0} H_{\text {cub }}$ is $-48 \mathrm{mT}$. We observe that the magnetic damping constant is the same along different crystalline directions with a value of $\alpha_{\mathrm{Fe}}=0.0041 \pm 0.0004$. It is worth noting here that the frequency-independent contribution due to inhomogeneity is quite low, $\mu_{0} \Delta \mathrm{H}_{0} \cong 0.4 \mathrm{mT}$, which is another verification of high quality epitaxial growth.

In order to evaluate the charge-spin current conversion, spin-orbit ferromagnetic resonance (SO-FMR) was used. This is also known as spin transfer or spin-torque FMR (STFMR) or spin diode FMR, which is somehow the reciprocal dynamic effect of spin pumping FMR. We inject directly the microwave frequency charge current in the $s / / \mathrm{Fe} / \mathrm{Pt}$ slabs which is converted into spin current inside the Pt layer due to the SHE. Therefore, an oscillating spin current is injected from the Pt layer into the Fe layer inducing precession of its magnetization. This, in turn, leads to an oscillatory radiofrequency resistance which mixed with the rf current allows, at the resonance condition, dc voltage detection across the slab using a bias tee. ${ }^{6,21-24}$ The dc voltage is picked up at $45^{\circ}$ of the applied $H_{\mathrm{dc}}$ (see insets in Fig. 3). The dc voltage is composed of a mix between a symmetrical Lorentzian function and an antisymmetrical one around the resonance field $H_{\text {res }}$. The amplitude of each contribution is $V_{\text {sym }}$ and $V_{\text {anti. }}$. We use

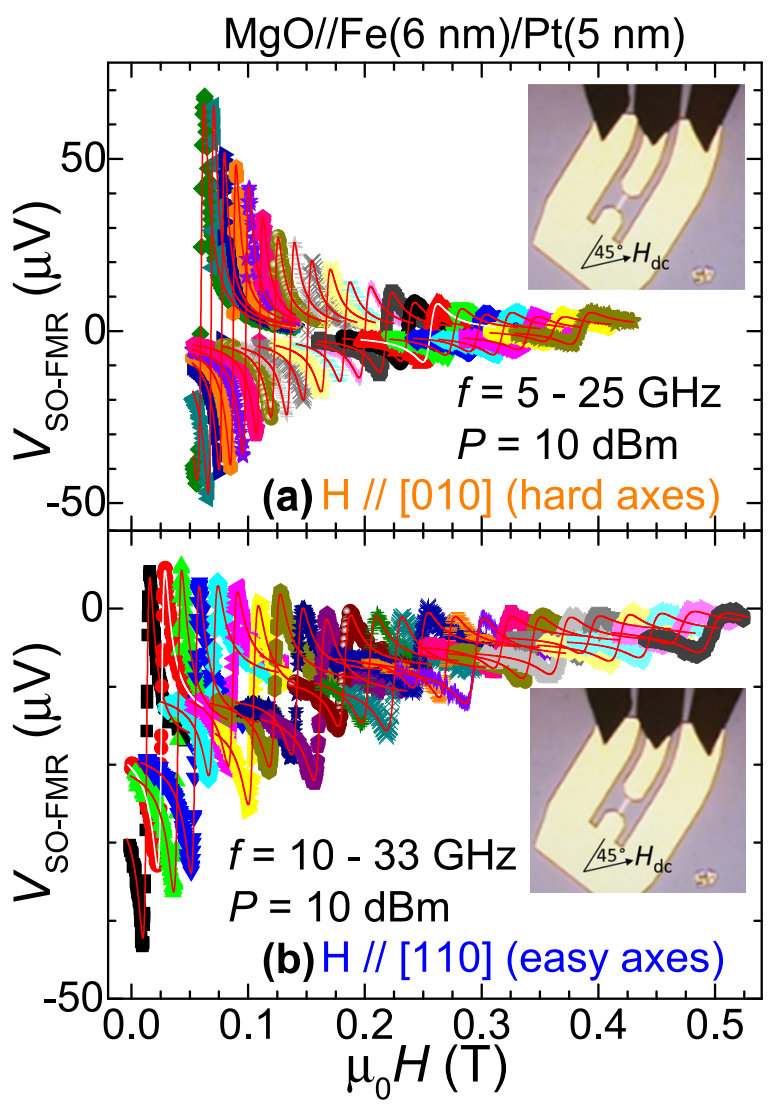

FIG. 3. Raw data of the SO-FMR scans. (a) $H_{\mathrm{dc}}$ is applied parallel to the [010] $\mathrm{MgO}$ crystalline direction (scans shown only for $\mathrm{f} \geq 5 \mathrm{GHz}$ ). (b) $H_{\mathrm{dc}}$ is applied parallel to the $[110] \mathrm{MgO}$ crystalline direction. Insets are pictures of devices along with Ti/Au electrodes and GSG rf contacts. The slabs were patterned at different crystalline directions. $H_{\mathrm{dc}}$ is always applied at $45^{\circ}$ of the slab. 
the following general function to fit the mixed voltage measured considering also an offset $\mathrm{V}_{\text {offset }}$ :

$$
\begin{aligned}
V_{\text {mix }}= & V_{\text {offset }}+V_{\text {sym }} \frac{\Delta H^{2}}{\Delta H^{2}+\left(H-H_{r e s}\right)^{2}} \\
& +V_{\text {anti }} \frac{\left(H-H_{r e s}\right) \Delta H}{\Delta H^{2}+\left(H-H_{r e s}\right)^{2}} .
\end{aligned}
$$

The slabs for SO-FMR were patterned along different crystallographic directions by standard UV lithography and have lateral sizes of $20 \mu \mathrm{m} \times 90 \mu \mathrm{m}$. Ti/Au electrodes were deposited by the lift off technique. In the inset of Fig. 3(a), a picture of one device along with grounded-signal-grounded, GSG, rf contacts is shown. Figure 3 shows raw data of the dc voltage measured when $H_{d c}$ is applied along different crystalline axes. After the analysis of this broadband frequency dependence result, we show that by SO-FMR we can also account for the in-plane magnetic anisotropies, i.e., the easy (hard) axes when $H_{\mathrm{dc}}$ is parallel to [110]MgO $([100] \mathrm{MgO})$, which is summarized in Fig. 4(a) where we can identify similar results of only FMR as observed in Fig. 2(c). This means that despite the fact that the pick-up voltage is at $45^{\circ}$ of the applied field in the SO-FMR experiment, what matters is the direction along which $H_{\mathrm{dc}}$ is applied with respect to the crystalline axis of the samples. Thus, this technique also allows us to study magnetic anisotropies not only in polycrystalline or amorphous samples but also in epitaxial samples. The magnetic damping in $\mathrm{Fe} / \mathrm{Pt}$ results in $\alpha_{\mathrm{Fe} / \mathrm{Pt}}=0.0081 \pm 0.0008$ as shown in Fig. 4(b). We note that this is one of the lowest values of total magnetic damping reported in a metallic magnetic/Pt bilayer. This value is even comparable to the one reported in sputtered $\mathrm{CoFeB} / \mathrm{Ta}, 0.008^{9}$ or lower than the one reported in $\mathrm{NiFe} / \mathrm{Ta}$, above $0.010 .{ }^{29} \mathrm{With}$ the results from the Fe reference layer $\left(\alpha_{\mathrm{Fe}}=0.0041 \pm 0.0004\right)$, we can calculate the effective spin mixing conductance $g_{\text {eff }}^{\uparrow \uparrow}$ following the standard spin pumping theory $8,13,30$

$$
g_{\text {eff }}^{\uparrow \downarrow}=\frac{4 \pi M_{\mathrm{s}} t_{\mathrm{Fe}}}{g \mu_{\mathrm{B}}}\left(\alpha_{\mathrm{Fe} / \mathrm{Pt}}-\alpha_{\mathrm{Fe}}\right),
$$

where $\alpha_{\mathrm{Fe}}$ is quite large. To compute $g_{\text {eff }}^{\uparrow \downarrow}$, we use the difference of the magnetic damping $\left(\alpha_{\mathrm{Fe} / \mathrm{Pt}}-\alpha_{\mathrm{Fe}}\right)$ which gives the contribution due to spin pumping only since the Fe layer is a true reference sample as described above.

This results in $g_{\text {effect }}^{\uparrow \downarrow}=2.6 \pm 0.5 \times 10^{19}$ which is in the low range of previously reported values for FM/Pt systems and similar to epitaxial Fe/Pt samples studied by spin pumping. ${ }^{31,32}$ Critical for this result is that the Fe layer is exactly the same for both reference $\mathrm{Fe} / \mathrm{MgO}$ and $\mathrm{Fe} / \mathrm{Pt}$ bilayers which is crucial especially in epitaxial samples where damping is much more sensitive to any defect (which is not the case in polycrystalline samples). Future work will include measuring the reference damping with the same SO-FMR method using a structure like $\mathrm{Fe} / \mathrm{Cu} / \mathrm{MgO}$ and thickness dependence.

In the simplest model, the quantification of the effective spin Hall angle $\theta_{\mathrm{SHE}}^{\text {effect }}$ is proportional to the ratio of the symmetrical Lorentzian voltage over the anti-Lorentzian one, $\mathrm{V}_{\text {symm }} / \mathrm{V}_{\text {anti. }}$. When the resonance field is large enough to consider uniform precession of the magnetization, we can use the simplest model to calculate the effective spin Hall angle ${ }^{6,22-24}$
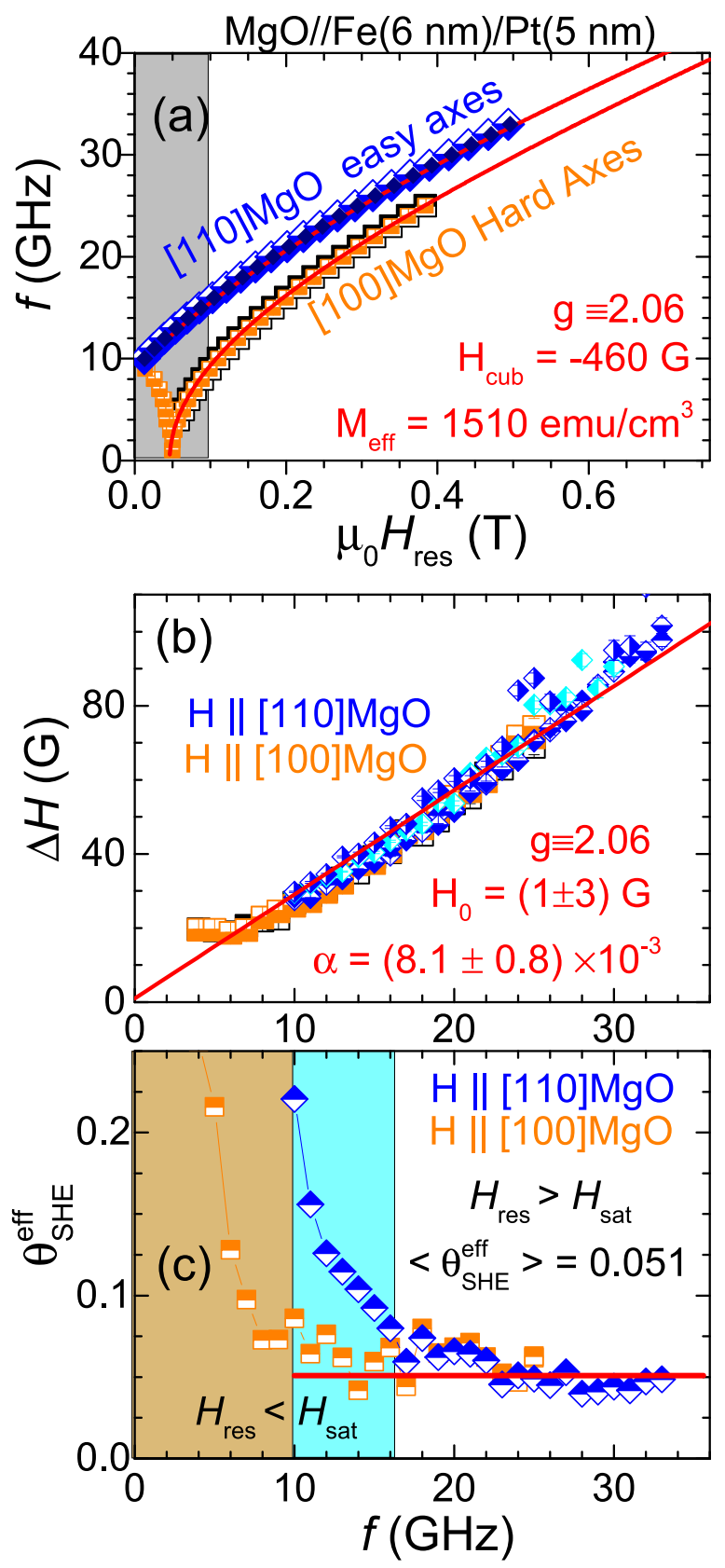

FIG. 4. Results of the SO-FMR study. (a) Dispersion relationship and magnetic anisotropy determination. The grey shadowed region stands for the condition $H_{\text {res }}<H_{\text {sat }}$ (b) Linewidth and damping constant determination. (c) Determination of the effective spin Hall angle according to Eq. (3) which is reliable only when $H_{\text {res }}>H_{\text {sat }}$ (red line stands for the average). We observe that for $\mathrm{f}>10 \mathrm{GHz}$ ( $>15 \mathrm{GHz}$ ) along the hard (easy) axes, it reaches a constant value (as expected it does not depend on the frequency). The resonance field for such a frequency is above $0.1 \mathrm{~T}$, i.e., well above saturation field so uniform precession of magnetization is reached. The shadows regions stand for the condition $H_{\text {res }}<H_{\text {sat }}$ as in (a).

$$
\text { If } \begin{aligned}
H_{\mathrm{sat}} & <H_{\mathrm{res}} \rightarrow \theta_{\mathrm{SHE}}^{e f f}=\frac{J_{\mathrm{s}}}{J_{\mathrm{c}}} \\
& \cong \frac{V_{\mathrm{sym}}}{V_{\text {anti }}} \frac{e \mu_{0} M_{\mathrm{s}} t_{\mathrm{Fe}} t_{\mathrm{Pt}}}{\hbar}\left[1+\frac{4 \pi M_{\mathrm{eff}}}{H_{\mathrm{res}}}\right]^{1 / 2},
\end{aligned}
$$

where we have highlighted the condition of uniform precession of the magnetization $\left(H_{\text {res }}>H_{\text {sat }}\right)$, with $H_{\text {sat }}$ being the saturation field. In the in-plane hard axes, the field necessary to get the saturation state is above $0.1 \mathrm{~T}$, obtained after $M(\mathrm{H})$ 
cycles in PPMS-VSM (not shown). The saturation magnetization is $M_{\mathrm{s}}=1675 \mathrm{emu} / \mathrm{cm}^{3}$, close to the bulk value $\left(1714 \mathrm{emu} / \mathrm{cm}^{3}\right) .^{33} \mathrm{We}$ can calculate the four-fold magnetocrystalline anisotropy $\left(K_{\text {cub }}=H_{\text {cub }} \times M_{\mathrm{s}} / 2\right)$. It results in our Fe film that $K_{\text {cub }} \approx 3.9 \times 10^{5} \mathrm{erg} / \mathrm{cm}^{3}$, below the bulk value of $\mathrm{Fe}\left(\approx 4.8 \times 10^{5} \mathrm{erg} / \mathrm{cm}^{3}\right) .{ }^{33}$ As shown in Fig. 4(c) and using Eq. (3), we find that for $\mathrm{Pt}$ in the $\mathrm{Fe} / \mathrm{Pt}$ system $\theta_{\mathrm{SHE}}^{\text {effect }}=0.051 \pm 0.005$, which is similar to previous effective values reported. ${ }^{6,8}$ Moreover, within the experimental resolution, we have found similar values when $H$ is applied along different crystalline directions once it reaches the condition: $H_{\text {res }}>H_{\text {sat }}$ as displayed in Fig. 4(c).

Additionally, we have grown $s / / \mathrm{Fe}(6) / \mathrm{Pt}(t)$ with $t$ between 1 and $10 \mathrm{~nm}$ and then patterned in a double Hall bar to measure the electrical resistance. From the Pt-thickness dependence of such results, we have estimated the resistivity of the Pt layer whose value is $\rho_{\mathrm{Pt}}=15.6 \pm 0.5 \mu \Omega \mathrm{cm}$ along different in-plane crystalline directions. It has been shown that the Elliott-Yafet mechanism dominates the conduction electron spin relaxation in $\mathrm{Pt}^{15,34,35}$ where the product $\rho_{\mathrm{Pt}}$ $\times l_{\mathrm{sf}}$ is a constant. Experimental values of such a product ${ }^{8,34}$ are close to the theoretical one, $\rho_{\mathrm{Pt}} \times l_{\mathrm{sf}} \approx 0.61 f \Omega \mathrm{m}^{2}{ }^{15}$ Thus, we can consider that in this epitaxial system, the spin diffusion length of $\mathrm{Pt}$ is $l_{\mathrm{sf}} \approx 3.9 \mathrm{~nm}$. The estimated product $\theta_{\mathrm{SHE}}^{\text {effect }} \times l s f \approx 0.19 \mathrm{~nm}$ results in the value close to that reported in most studies where Pt-thickness dependence was studied. $^{8}$

Some other systems that showed isotropic results despite in-plane anisotropies can be found in the case of in-plane exchange bias and spin-orbit torque in antiferromagnetic/ ferromagnetic $(\mathrm{AFM} / \mathrm{FM})^{36}$ or isotropic damping in epitaxial $\mathrm{Fe},{ }^{37}$ for a similar level of thicknesses. However, it has just been shown that for an ultrathin Fe layer $(0.8 \mathrm{~nm})$, we can detect anisotropic magnetic damping, ${ }^{37}$ as predicted theoretically for epitaxial magnetic layers. ${ }^{38}$ Ultrathin magnetic layers are out of the scope of the present study, but it is a very interesting perspective to enhance this study especially with epitaxial Heusler alloys showing ultralow damping. ${ }^{39}$

The intensity of the FMR spectrum is proportional to the intensity of the radiofrequency field $h_{\mathrm{rf}}$ and the one of SOFMR scan is proportional to $h_{\mathrm{rf}}^{2}$. This radiofrequency field affects the amplitude but not the line-shape of FMR or SOFMR curves in the magnetization linear response regime (low microwave power). Thus, the results shown in the present study, Figs. 2(c), 2(d), and 4, are correct even if $h_{\mathrm{rf}}$ is anisotropic due to the monocrystalline $\mathrm{MgO}$ substrate. Similarly, the anisotropic magnetoresistance (AMR) affects the amplitude but not the shape of SO-FMR scans. SHA quantification using Eq. (3) cancels $h_{\mathrm{rf}}$ amplitude and AMR factor as shown in Ref. 6.

In summary, we have performed FMR and SO-FMR in thin highly epitaxial $\mathrm{Fe}$ and $\mathrm{Fe} / \mathrm{Pt}$ samples, respectively. From both methods, we show that one can access detailed magnetic anisotropies. Particularly, we have accounted for the cubic magnetocrystalline anisotropy of Fe. Using exactly the same Fe bottom layer, a low value of spin mixing conductance in $\mathrm{Fe} / \mathrm{Pt}$ was measured. We show that considering uniform precession of the magnetization (resonance field above the saturation field), the effective spin Hall angle can be determined in a reliable way for FM/Pt systems, and it is independent of the microwave frequency. Furthermore, we show that the magnetic Gilbert damping constant and the effective spin Hall angle are isotropic in the epitaxial system for the level of thickness in the present study, $\mathrm{Fe}(6 \mathrm{~nm}) /$ $\operatorname{Pt}(5 \mathrm{~nm})$.

Our results also highlight the importance of taking care of the proper conditions for the estimation of the effective charge-spin current conversion efficiency or spin Hall angle and the effective spin mixing conductance in epitaxial FM/ HM systems. Those parameters and their proper determination are relevant for spintronic applications.

This work was supported partly by the French PIA project "Lorraine Université d'Excellence," reference ANR15-IDEX-04-LUE. By the ANR-NSF Project, ANR-13-IS040008-01. Experiments were performed using equipment from the TUBE-Daum funded by FEDER (EU), ANR, the Region Lorraine and Grand Nancy. We thank L. E. Ocola for a proof reading of the manuscript.

${ }^{1}$ M. I. Dyakonov and V. I. Perel, "Current-induced spin orientation of electrons in semiconductors," Phys. Lett. A 35, 459 (1971).

2J. E. Hirsch, "Spin Hall effect," Phys. Rev. Lett. 83, 1834 (1999).

${ }^{3}$ A. Hoffmann, "Spin Hall effects in metals," IEEE Trans. Magn. 49, 5172 (2013).

${ }^{4} J$. Sinova, S. O. Valenzuela, J. Wunderlich, C. H. Back, and T. Jungwirth, “Spin Hall effects," Rev. Mod. Phys. 87, 1213 (2015).

${ }^{5}$ L. Vila, T. Kimura, and Y. Otani, "Evolution of the spin Hall effect in Pt nanowires: Size and temperature effects," Phys. Rev. Lett. 99, 226604 (2007).

${ }^{6}$ L. Liu, T. Moriyama, D. C. Ralph, and R. A. Buhrman, "Spin-torque ferromagnetic resonance induced by the spin Hall effect," Phys. Rev. Lett. 106, 036601 (2011).

${ }^{7}$ A. Azevedo, L. H. Vilela-Leão, R. L. Rodríguez-Suárez, A. F. Lacerda Santos, and S. M. Rezende, "Spin pumping and anisotropic magnetoresistance voltages in magnetic bilayers: Theory and experiment," Phys. Rev. B 83, 144402 (2011).

${ }^{8}$ J. C. Rojas-Sánchez, N. Reyren, P. Laczkowski, W. Savero, J. P. Attané, C. Deranlot, M. Jamet, J. M. George, L. Vila, and H. Jaffrès, "Spin pumping and inverse spin Hall effect in platinum: The essential role of spinmemory loss at metallic interfaces," Phys. Rev. Lett. 112, 106602 (2014).

${ }^{9}$ L. Liu, C.-F. Pai, Y. Li, H. W. Tseng, D. C. Ralph, and R. A. Buhrman, "Spin-torque switching with the giant spin Hall effect of tantalum," Science 336, 555 (2012).

${ }^{10}$ C.-F. Pai, L. Liu, Y. Li, H. W. Tseng, D. C. Ralph, and R. A. Buhrman, "Spin transfer torque devices utilizing the giant spin Hall effect of tungsten,” Appl. Phys. Lett. 101, 122404 (2012).

${ }^{11}$ J. Torrejon, J. Kim, J. Sinha, S. Mitani, M. Hayashi, M. Yamanouchi, and $\mathrm{H}$. Ohno, "Interface control of the magnetic chirality in $\mathrm{CoFeB} / \mathrm{MgO}$ heterostructures with heavy-metal underlayers," Nat. Commun. 5, 4655 (2014).

${ }^{12}$ Y. Niimi, Y. Kawanishi, D. H. Wei, C. Deranlot, H. X. Yang, M. Chshiev, T. Valet, A. Fert, and Y. Otani, "Giant spin Hall effect induced by skew scattering from bismuth impurities inside thin film CuBi alloys," Phys. Rev. Lett. 109, 156602 (2012).

${ }^{13}$ P. Laczkowski, J. C. Rojas-Sánchez, W. Savero-Torres, H. Jaffrès, N. Reyren, C. Deranlot, L. Notin, C. Beigné, A. Marty, J. P. Attané, L. Vila, J. M. George, and A. Fert, "Experimental evidences of a large extrinsic spin Hall effect in AuW alloy,” Appl. Phys. Lett. 104, 142403 (2014).

${ }^{14}$ P. Laczkowski, Y. Fu, H. Yang, J.-C. Rojas-Sánchez, P. Noel, V. T. Pham, G. Zahnd, C. Deranlot, S. Collin, C. Bouard, P. Warin, V. Maurel, M. Chshiev, A. Marty, J.-P. Attané, A. Fert, H. Jaffrès, L. Vila, and J.-M. George, "Large enhancement of the spin Hall effect in Au by side-jump scattering on Ta impurities," Phys. Rev. B 96, 140405(R) (2017).

${ }^{15}$ Y. Liu, Z. Yuan, R. J. H. Wesselink, A. A. Starikov, and P. J. Kelly, "Interface enhancement of gilbert damping from first principles," Phys. Rev. Lett. 113, 207202 (2014).

${ }^{16} \mathrm{~K}$. Dolui and B. K. Nikolic, "Spin-memory loss due to spin-orbit coupling at ferromagnet/heavy-metal interfaces: Ab initio spin-density matrix approach,” Phys. Rev. B 96, 220403 (2017). 
${ }^{17}$ A. Azevedo, L. H. Vilela Leão, R. L. Rodriguez-Suarez, A. B. Oliveira, and S. M. Rezende, "Dc effect in ferromagnetic resonance: Evidence of the spin-pumping effect?,"J. Appl. Phys. 97, 10C715 (2005).

${ }^{18}$ E. Saitoh, M. Ueda, H. Miyajima, and G. Tatara, "Conversion of spin current into charge current at room temperature: Inverse spin-Hall effect," Appl. Phys. Lett. 88, 182509 (2006).

${ }^{19}$ H. Nakayama, K. Ando, K. Harii, T. Yoshino, R. Takahashi, Y. Kajiwara, K. Uchida, Y. Fujikawa, and E. Saitoh, "Geometry dependence on inverse spin Hall effect induced by spin pumping in $\mathrm{Ni}_{81} \mathrm{Fe}_{19} / \mathrm{Pt}$ films," Phys. Rev. B 85, 144408 (2012).

${ }^{20}$ O. Mosendz, V. Vlaminck, J. E. Pearson, F. Y. Fradin, G. E. W. Bauer, S. D. Bader, and A. Hoffmann, "Detection and quantification of inverse spin Hall effect from spin pumping in permalloy/normal metal bilayers," Phys. Rev. B 82, 214403 (2010).

${ }^{21}$ D. Fang, H. Kurebayashi, J. Wunderlich, K. Vyborny, L. P. Zarbo, R. P. Campion, A. Casiraghi, B. L. Gallagher, T. Jungwirth, and A. J. Ferguson, "Spin-orbit-driven ferromagnetic resonance," Nat. Nanotechnol. 6, 413 (2011).

${ }^{22}$ K. Kondou, H. Sukegawa, S. Mitani, K. Tsukagoshi, and S. Kasai, "Evaluation of spin Hall angle and spin diffusion length by using spin current-induced ferromagnetic resonance," Appl. Phys. Express 5, 073002 (2012).

${ }^{23}$ A. Ganguly, K. Kondou, H. Sukegawa, S. Mitani, S. Kasai, Y. Niimi, Y. Otani, and A. Barman, "Thickness dependence of spin torque ferromagnetic resonance in $\mathrm{Co}_{75} \mathrm{Fe}_{25} / \mathrm{Pt}$ bilayer films," Appl. Phys. Lett. 104, 072405 (2014).

${ }^{24}$ V. Tshitoyan, C. Ciccarelli, A. P. Mihai, M. Ali, A. C. Irvine, T. A. Moore, T. Jungwirth, and A. J. Ferguson, "Electrical manipulation of ferromagnetic NiFe by antiferromagnetic IrMn,” Phys. Rev. B 98, 214406 (2015).

${ }^{25}$ O. Kohmoto and C. Alexander, Jr., "Ferromagnetic resonance in epitaxial Fe films on MgO,” Jpn. J. Appl. Phys. Part I 31, 2101 (1992).

${ }^{26} \mathrm{~J}$. Smit and H. G. Beljers, "Ferromagnetic resonance absorption in $\mathrm{BaFe}_{12} \mathrm{O}_{19}$, a highly anisotropic crystal," Philips Res. Rep. 10, 113 (1955).

${ }^{27} \mathrm{H}$. Suhl, "Ferromagnetic resonance in nickel ferrite between one and two kilomegacycles,” Phys. Rev. 97, 555 (1955).

${ }^{28}$ J.-C. Rojas-Sánchez, M. Cubukcu, A. Jain, C. Vergnaud, C. Portemont, C. Ducruet, A. Barski, A. Marty, L. Vila, J. P. Attané, E. Augendre, G.
Desfonds, S. Gambarelli, H. Jaffrès, J. M. George, and M. Jamet, "Spin pumping and inverse spin Hall effect in germanium," Phys. Rev. B 88, 064403 (2013).

${ }^{29}$ J. E. Gomez, B. Zerai Tedlla, N. R. Alvarez, G. Alejandro, E. Goovaerts, and A. Butera, "Spin transport parameters in $\mathrm{Ni}_{80} \mathrm{Fe}_{20} / \mathrm{Ru}$ and $\mathrm{Ni}_{80} \mathrm{Fe}_{20} / \mathrm{Ta}$ bilayers," Phys. Rev. B 90, 184401 (2014).

${ }^{30}$ Y. Tserkovnyak, A. Brataas, and B. I. Halperin, "Nonlocal magnetization dynamics in ferromagnetic heterostructures," Rev. Mod. Phys. 77, 1375 (2005).

${ }^{31}$ E. T. Papaioannou, P. Fuhrmann, M. B. Jungfleisch, T. Brächer, P. Pirro, V. Lauer, J. Lösch, and B. Hillebrands, "Pt bilayers optimizing the spinpumping induced inverse spin Hall voltage by crystal growth in $\mathrm{Fe} / \mathrm{Pt}$ bilayers," Appl. Phys. Lett. 103, 162401 (2013).

${ }^{32}$ A. Conca, S. Keller, L. Mihalceanu, T. Kehagias, G. P. Dimitrakopulos, B. Hillebrands, and E. T. Papaioannou, "Study of fully epitaxial Fe/Pt bilayers for spin pumping by ferromagnetic resonance spectroscopy," Phys. Rev. B 93, 134405 (2016).

${ }^{33}$ B. D. Cullity and C. D. Graham, Introduction to Magnetic Materials, 2nd ed. (Jonh Wiley \& Sons, Inc., New Jersey, 2009).

${ }^{34}$ E. Sagasta, Y. Omori, M. Isasa, M. Gradhand, L. E. Hueso, Y. Niimi, and Y. Otani, "Tuning the spin Hall effect of Pt from the moderately dirty to the superclean regime," Phys. Rev. B 94, 060412 (2016).

${ }^{35}$ M. Nguyen, D. C. Ralph, and R. A. Buhrman, "Spin torque study of the spin Hall conductivity and spin diffusion length in platinum thin films with varying resistivity," Phys. Rev. Lett. 116, 126601 (2016).

${ }^{36}$ H. Saglam, J. C. Rojas-sanchez, S. Petit, M. Hehn, W. Zhang, J. E. Pearson, S. Mangin, and A. Hoffmann, "Independence of spin-orbit torques from the exchange bias direction in $\mathrm{Ni}_{81} \mathrm{Fe}_{19} / \mathrm{IrMn}$ bilayers," Phys. Rev. B 98, 094407 (2018).

${ }^{37}$ L. Chen, S. Mankovsky, S. Wimmer, M. A. W. Schoen, H. S. Körner, M. Kronseder, D. Schuh, D. Bougeard, H. Ebert, D. Weiss, and C. H. Back, "Emergence of anisotropic Gilbert damping in ultrathin Fe layers on GaAs (001)," Nat. Phys. 14, 490 (2018).

${ }^{38}$ K. Gilmore, M. D. Stiles, J. Seib, D. Steiauf, and M. Fähnle, "Anisotropic damping of the magnetization dynamics in Ni, Co, and Fe," Phys. Rev. B 81, 174414 (2010).

${ }^{39}$ S. Andrieu, A. Neggache, T. Hauet, T. Devolder, A. Hallal, M. Chschiev, A. Bataille, P. L. Fevre, and F. Bertran, "Direct evidence for minority spin gap in the $\mathrm{Co}_{2} \mathrm{MnSi}$ Heusler alloy," Phys. Rev. B 93, 094417 (2016). 